\title{
Percepción y prevención de riesgos ocupacionales en veterinarios rurales
}

\author{
Tarabla, H.D. ${ }^{1}$; Hernández, A.C. ${ }^{1}$; Molineri, A.I. ${ }^{2}$, Signorini, M.L. ${ }^{1,2}$ \\ ${ }^{1}$ Facultad de Ciencias Veterinarias, Univ. Nac. Litoral, Kreder 2805, Esperanza (3080), Santa Fe, Argentina, \\ Tel. 03496-420639. ${ }^{2}$ CONICET, INTA EEA-Rafaela, Santa Fe, Argentina. \\ E-mail: amolineri83@gmail.com
}

\begin{abstract}
Resumen
Tarabla, H.D.; Hernández, A.C.; Molineri, A.I.; Signorini, M.L.: Percepción y prevención de riesgos ocupacionales en veterinarios rurales. Rev. vet. 28: 2, 152-156, 2017. El ejercicio de la profesión veterinaria está asociado a numerosos riesgos ocupacionales. Los objetivos de este trabajo fueron evaluar la percepción de riesgos laborales (PRL), el uso de elementos de protección personal (EPP) y las asociaciones entre sí y con variables sociodemográficas en veterinarios rurales de la Provincia de Santa Fe (Argentina). Se desarrolló un estudio transversal encuestando a 562 veterinarios. Aunque las PRL tendieron a estar correlacionadas entre sí, los coeficientes fueron relativamente bajos. Los guantes fueron los EPP más utilizados, pero la frecuencia de uso de otros elementos y la utilización de más de un EPP para una misma actividad fueron bajas. El uso de EPP fue independiente de la PRL. Tanto PRL como uso de EPP estuvieron asociados al sexo y la edad de los encuestados. La falta de uso de distintos EPP en forma conjunta indica una real inconsistencia en el comportamiento profesional de autoprotección.
\end{abstract}

Palabras clave: veterinaria, riesgos ocupacionales, percepción de riesgos, elementos de protección personal.

\begin{abstract}
Tarabla, H.D.; Hernández, A.C.; Molineri, A.I.; Signorini, M.L.: Perception and prevention of occupational risks in rural veterinarians. Rev. vet. 28: 2, 152-156, 2017. Veterinary practice is associated with many occupational hazards. The objectives of this study were to evaluate the occupational risk perception (ORP), use of personal protective equipment (PPE), and the associations between them and with socio-demographic characteristics of rural veterinarians of the Province of Santa Fe (Argentina). A cross-sectional study was carried out on $n=562$ veterinarians by means of a structured questionnaire. Although ORP tended to be correlated with each other, the coefficients were relatively low. Gloves were the most frequently used PPE, but other element usage was scarce. The use of PPE in conjunction in a single activity was low. PPE usage was independent of the ORP. Both ORP and use of PPE were associated with respondents' sex and age. The lack of use of PPE in conjunction indicates a real inconsistency in the professional behavior of self-protection.
\end{abstract}

Key words: veterinary practice, occupational risks, risk perception, personal protective equipment.

\section{INTRODUCCIÓN}

El ejercicio de la medicina veterinaria está asociado a accidentes laborales y enfermedades profesionales. En América Latina los accidentes in labore están relacionados principalmente con heridas punzo-cortantes, mordeduras, rasguños, atropellamientos y aprisionamientos por animales ${ }^{1,8}$, mientras que las enfermedades profesionales se relacionan básicamente con zoonosis y trastornos ergonómicos 5,20 .

Aunque la mayoría de las lesiones relacionadas con el trabajo pueden prevenirse mediante la adopción de

Recibido: 24 agosto 2016 / Aceptado: 13 febrero 2017 medidas de seguridad y uso de elementos de protección personal (EPP) ${ }^{3,13,17}$, las relaciones entre la conciencia de un peligro relacionado con el trabajo, la percepción de riesgos laborales (PRL) y la adopción de medidas de seguridad, no ha sido claramente establecida.

Las prácticas preventivas y las frecuencias de uso de EPP varían en las distintas actividades profesionales y podrían estar influenciadas por las percepciones, las políticas y las culturas instaladas en los lugares de trabajo ${ }^{10}$.

Los objetivos de esta comunicación fueron evaluar la PRL, la frecuencia de uso de EPP y las asociaciones entre sí y con variables socio-demográficas en veterinarios rurales de la Provincia de Santa Fe, Argentina. 


\section{MATERIAL Y MÉTODOS}

Se efectuó un estudio observacional sobre PRL y medidas preventivas tomadas por profesionales veterinarios que se dedicaban al manejo y atención de grandes animales en la región señalada ut supra. El diseño fue de tipo transversal, la unidad de interés fue el veterinario y la población-objetivo fueron los veterinarios rurales santafesinos.

En dicha zona, los profesionales actúan como coresponsables sanitarios en planes de control de enfermedades notificables y participaron en jornadas obligatorias de capacitación continua programadas por el Colegio de Veterinarios local. En las mismas se censaron 741 profesionales, de los cuales el 75,8\% $(n=562)$ respondieron a un cuestionario estructurado. Esto se correspondió con un error absoluto del 2,5\%, un nivel de confianza del $99 \%$ y una frecuencia esperada del evento del $70 \%$.

El cuestionario estuvo dividido en tres secciones: a) características demográficas del entrevistado, b) PRL a los cuales se encuentran expuestos y c) EPP utilizados en la práctica profesional. Previamente a la distribución del cuestionario, se explicó la finalidad e importancia de la encuesta, poniéndose énfasis en que el interés de las mismas no estaba en las actividades o actitudes de ningún colega en particular, sino en las frecuencias a nivel poblacional, por lo que las respuestas debían ser anónimas.

"Percepción del riesgo" fue definida como la noción de la probabilidad que una actividad pueda causar daño en la salud o integridad física del profesional, de acuerdo a lo establecido por la Organización Mundial de la Salud ${ }^{15}$. Esta probabilidad fue estimada de acuerdo a una escala ordinal (alta/media/ baja). La frecuencia de uso de EPP se estimó mediante una escala ordinal (nunca/ a veces/ siempre) utilizada en trabajos previos de los autores de esta comunicación.

Se consideraron cuatro características demográficas de los encuestados para relacionarlas con la percepción de los riesgos y el uso de elementos de protección: edad, sexo, antigüedad profesional y región geográfica en donde desempeña su actividad. El análisis estadístico incluyó $t$ de Student, ANOVA, $\chi^{2}$ y correlaciones de Pearson y Spearman.

\section{RESULTADOS}

En promedio, los entrevistados tenían 42,2 $\pm 10,1$ años de edad y habían desarrollado su actividad profesional a lo largo de 16,2 $\pm 11,1$ años. El 93,1\% eran hombres y $6,9 \%$ mujeres, quienes eran más jóvenes y con menos años de graduadas que los primeros $(p<0,0001)$. Edad y antigüedad profesional estuvieron significativamente asociadas $(\mathrm{p}<0,0001)$. La región geográfica donde se desarrollaba la labor profesional no estuvo significativamente asociada a ninguna de las otras variables sociodemográficas.

Sólo el $25,9 \%$ de los encuestados se había efectuado un análisis de brucelosis hacía menos de un año, el $18,4 \%$ entre uno y dos años y el 55,7\% hacía más de dos años, mientras que se habían efectuado una revisión médica de rutina en esos períodos el 47,5; 22,4; y $30,2 \%$ respectivamente. El tiempo transcurrido desde la última vacunación antitetánica superó los 10 años en el $28,8 \%$ de los encuestados.

Percepción de riesgos. Entre las tareas profesionales, la más alta percepción del riesgo se observó en la manipulación de abortos, como indica la Figura 1.

La vacunación antibrucélica fue considerada más riesgosa por las mujeres que por los hombres $(\mathrm{p}<0,05)$. Por su parte, la mayor cantidad de años de experiencia profesional correlacionó con las menores percepciones de riesgos en la atención de partos y vacunación antibrucélica $(p<0,01)$. Los grados de riesgo percibidos en las actividades profesionales tendieron a estar correlacionados entre sí, pero los coeficientes de correlación no superaron el coeficiente 0,5 (Tabla 1 ). No se encontraron asociaciones entre PRL y región geográfica de ejercicio profesional.

Uso de elementos de protección personal. El uso de guantes tendió a ser más frecuente que el uso de otros

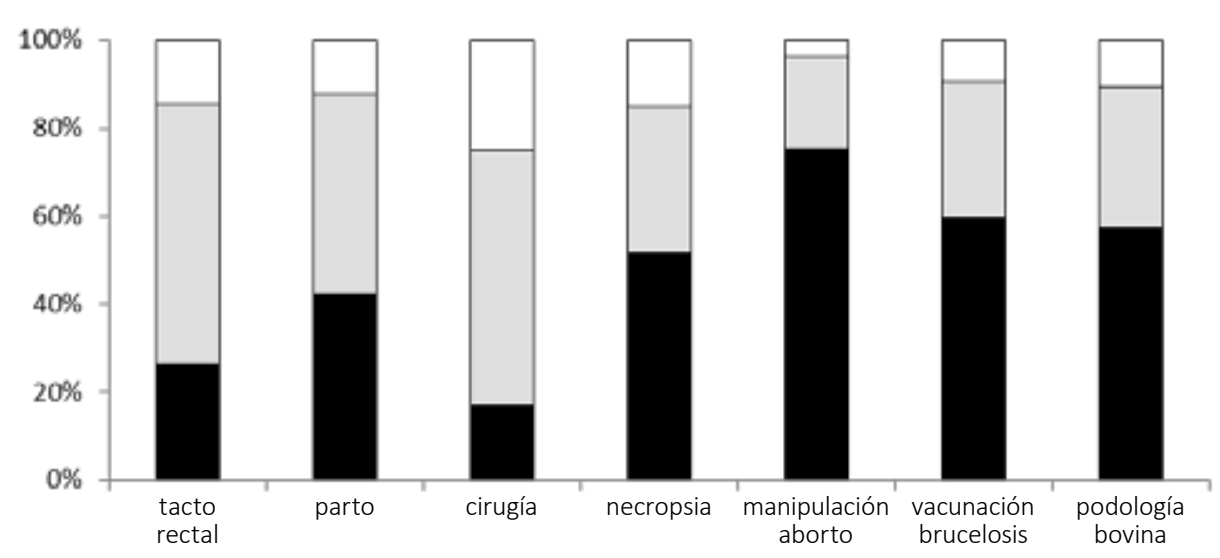

Figura 1. Percepción de riesgos laborales de veterinarios rurales con accidentes de trabajo de acuerdo al elemento involucrado (\%). Negro: alto, gris: medio, blanco: bajo. 
Tabla 1. Coeficientes de correlación de Spearman entre las percepciones de riesgo (bajo/medio/alto) en actividades profesionales.

\begin{tabular}{lcccccc}
\hline actividad & parto & cirugía & necropsia & aborto & VAB & podología \\
\hline tacto & $0,25^{*}$ & $0,21^{*}$ & $0,16^{*}$ & $0,17^{*}$ & 0,12 & $0,23^{*}$ \\
parto & - & $0,31^{*}$ & $0,48^{*}$ & $0,38^{*}$ & $0,26^{*}$ & $0,09^{*}$ \\
cirugía & - & - & $0,34^{*}$ & $0,24^{*}$ & $0,18^{*}$ & $0,13^{*}$ \\
necropsia & - & - & - & $0,39^{*}$ & $0,20^{*}$ & $0,13^{*}$ \\
aborto & - & - & - & - & $0,33^{*}$ & 0,06 \\
VAB & - & - & - & - & - & $0,14^{*}$ \\
\hline
\end{tabular}

${ }^{*} \mathrm{p}<0,05$. VAB: vacunación antibrucélica.

Tabla 2. Frecuencia de uso de elementos de protección utilizados en actividades profesionales por veterinarios rurales (Santa Fe, 2008).

\begin{tabular}{llccc}
\hline \multirow{2}{*}{ elementos } & actividad (n) & \multicolumn{3}{c}{ frecuencia (\%) } \\
\cline { 3 - 5 } guantes & tacto rectal (543) & 92,8 & 5,5 & 1,7 \\
& atención de partos (517) & 64,0 & 24,6 & 11,4 \\
& cirugía (520) & 60,2 & 28,8 & 11,0 \\
& necropsia (540) & 85,7 & 12,6 & 1,7 \\
& manipulación de abortos (497) & 88,3 & 6,8 & 4,8 \\
& vacunación antibrucélica (522) & 33,5 & 19,2 & 47,3 \\
& podología bovina (403) & 27,3 & 27,5 & 45,2 \\
\hline protectores & tacto rectal (511) & 2,7 & 4,3 & 93,0 \\
oculares & atención de partos (500) & 2,6 & 5,0 & 92,4 \\
o faciales & cirugía (496) & 2,6 & 2,8 & 94,6 \\
& necropsia (504) & 3,6 & 6,9 & 89,5 \\
& manipulación de abortos (472) & 4,2 & 4,4 & 91,3 \\
& vacunación antibrucélica (506) & 4,3 & 3,6 & 91,9 \\
& podología bovina (450) & 10,0 & 8,9 & 81,1 \\
\hline faja anatómica & requiere esfuerzo físico (549) & 6,0 & 19,3 & 74,7 \\
\hline tapones para oídos & ruidosas (532) & 0,4 & 3,0 & 96,6 \\
\hline
\end{tabular}

Tabla 3. Coeficientes de correlación de Spearman entre las frecuencias de uso de guantes (nunca/ a veces/ siempre) en actividades profesionales.

\begin{tabular}{lcccccc}
\hline actividades & parto & cirugía & necropsia & aborto & VAB & podología \\
\hline tacto & $0,23^{*}$ & $0,25^{*}$ & $0,29^{*}$ & $0,27^{*}$ & 0,10 & $0,18^{*}$ \\
parto & - & $0,50^{*}$ & $0,29^{*}$ & $0,25^{*}$ & $0,38^{*}$ & $0,33^{*}$ \\
cirugía & - & - & $0,32^{*}$ & $0,30^{*}$ & $0,38^{*}$ & $0,39^{*}$ \\
necropsia & - & - & - & $0,60^{*}$ & 0,12 & $0,20^{*}$ \\
aborto & - & - & - & - & 0,08 & 0,13 \\
VAB & - & - & - & - & - & $0,44^{*}$ \\
\hline
\end{tabular}

${ }^{*} \mathrm{p}<0,05$. VAB: vacunación antibrucélica

Tabla 4. Coeficientes de correlación de Spearman entre las frecuencias de uso de máscaras faciales o anteojos protectores (nunca/ a veces/ siempre).

\begin{tabular}{llcccccc}
\hline elemento & actividad & parto & cirugía & necropsia & aborto & VAB & podología \\
\hline antiparras & tacto & $0,66^{* *}$ & $0,65^{* *}$ & $0,63^{* *}$ & $0,58^{* *}$ & $0,50^{* *}$ & $0,36^{* *}$ \\
& parto & - & $0,61^{* *}$ & $0,71^{* *}$ & $0,75^{* *}$ & $0,53^{* *}$ & $0,12 *$ \\
& cirugía & - & - & $0,63 * *$ & $0,58^{* *}$ & $0,48^{* *}$ & $0,29 * *$ \\
& necropsia & - & - & - & $0,75^{* *}$ & $0,51^{* *}$ & $0,05 \ldots$ \\
& aborto & - & - & - & - & $0,59 * *$ & $0,40^{* *}$ \\
& VAB & - & - & - & - & - & $0,37 * *$ \\
\hline
\end{tabular}

${ }^{*} \mathrm{p}<0,05 * * ; \mathrm{p}<0,01$. VAB: vacunación antibrucélica elementos de protección en el ejercicio de las actividades profesionales. Por el contrario, la frecuencia de uso de EPP imprescindibles en algunas tareas (p.ej. máscaras faciales o anteojos protectores en vacunaciones antibrucélicas) fue extremadamente bajo (Tabla 2).

La utilización de guantes fue más frecuente en las mujeres que en los hombres $(p<0,01)$. Por su parte, a mayor cantidad de años de experiencia profesional correspondio una menor frecuencia de uso de guantes en partos, cirugías, tactos rectales y vacunaciones antibrucélicas $(p<0,01)$. No se encontraron asociaciones entre uso de EPP y la región geográfica de ejercicio profesional.

Las correlaciones entre la utilización de guantes en las distintas intervenciones profesionales tendieron a ser significativas, pero con coeficientes relativamente bajos (Tabla 3). La única correlación con $r>0,5$ fue entre el uso de guantes en necropsias y en manipulación de abortos y estuvo asociada al uso frecuente de estos elementos en ambas actividades.

Como indica la Tabla 2, la frecuencia de utilización de máscaras faciales o anteojos protectores fue muy baja o nula. Esta carencia hizo que las frecuencias de uso de este EPP en las distintas actividades tendieran a estar correlacionadas entre sí (Tabla 4). Por último, las correlaciones entre el uso de distintos EPP para una misma actividad fueron escasas o inexistentes.

Asociaciones entre percepción de riesgos y uso de elementos de protección personal. El uso de EPP tendió a ser independiente o a estar correlacionado con coeficientes muy bajos con la PRL en dicha actividad. A modo de ejemplos, la correlación entre percepción de riesgos de realizar tacto rectal y el uso de protección ocular fue $\mathrm{r}=0,107$, mientras que entre la percepción de riesgos de atención de partos y el uso de guantes fue $r=0,154(\mathrm{p}<0,05)$.

\section{DISCUSIÓN}

La tasa de respuesta al cuestionario propuesto fue buena (76\%). Debido a la íntima asociación entre edad y antigüedad profesional, se utilizó sólo la primera de estas variables en la búsqueda de 
asociaciones con PRL y uso de EPP. Coincidentemente a lo comunicado previamente, más del $50 \%$ de los veterinarios no se había sometido a una revisión médica de rutina o no se había hecho análisis para detección brucelosis en los últimos 12 meses.

Percepción de riesgos laborales. La percepción de riesgos es una construcción social que opera en contextos culturales, grupos e instituciones particulares, dentro de los cuales pueden ser afectadas por las características individuales de sus integrantes ${ }^{6,7}$. En el presente caso, la atención clínica fue percibida por este grupo de profesionales como de riesgo medio o bajo, contrastando con el hecho que la mayor parte de los accidentes laborales se producen en dichas instancias.

Similares contradicciones se observaron entre otras percepciones y el peligro real de determinadas actividades y actitudes. La percepción del riesgo de tareas ciertamente riesgosas como las necropsias a campo, fueron calificadas como de riesgo alto por sólo la mitad de encuestados y el tacto rectal, actividad relacionada con la presentación de patologías crónicas en miembros superiores ${ }^{1}$, fue considerada como de riesgo medio o bajo por tres de cada cuatro profesionales.

Observaciones semejantes fueron efectuadas en otras investigaciones. En profesionales de una región de Colombia, el 15\% de los encuestados consumía bebidas alcohólicas antes y durante el trabajo, pero sólo el $2 \%$ lo consideró un riesgo para la práctica profesional (López Cepeda et al., Salud Uninorte 30: 23-33). Por su parte, el riesgo del tránsito in itinere en rutas en la Provincia de Santa Fe fue considerado más alto que el tránsito en caminos rurales, pero la frecuencia real de accidentes es mayor en estos últimos.

A medida que aumentaron los años de experiencia profesional disminuyeron las PRL de algunas actividades profesionales. Al igual que en estudiantes de la carrera, las mujeres mostraron, en general, mayor PRL que los hombres, quienes tienden a minimizar los riesgos y a considerarlos menos problemáticos que las mujeres ${ }^{6,11,18}$.

Las percepciones de riesgo de las distintas actividades profesionales tendieron a estar correlacionadas entre sí. Sin embargo, aunque estas asociaciones fueron estadísticamente significativas, los coeficientes de correlación fueron relativamente bajos. Es probable que estas asociaciones estén más relacionadas con el alto número de encuestados que con una asociación real.

Uso de elementos de protección personal. Los guantes fueron los elementos más utilizados en las prácticas veterinarias. Sin embargo, el uso de otros EPP fue escaso. Aunque en algunos casos ocurren eventos imprevistos, por ejemplo rotura de los guantes al asistir bovinos durante el parto, no hay demasiadas razones -fuera de la imprudencia- que puedan explicar las razones por las cuales los guantes no hayan sido utilizados por todos los profesionales durante la realización de cirugías, necropsias y vacunaciones antibrucélicas con Cepa 19.

Similares observaciones se pueden hacer con respecto a la falta de uso de protectores oculares o faciales, más aún si se tiene en cuenta que la inoculación accidental y la exposición conjuntival son las formas más frecuentes de transmisión de brucelosis ${ }^{4,16}$, que es la enfermedad más prevalente en veterinarios de la región.

La mayor cantidad de años de experiencia profesional se correspondió con menor frecuencia de uso de EPP. Sin embargo, el riesgo de lesiones aumenta con la exposición acumulada y la experiencia no es un factor de protección en el ejercicio de la medicina veterinaria $8,12,14,19$.

Es interesante notar que esto puede variar en otras situaciones. En trabajos previos hemos comunicado que el uso de EPP fue más frecuente en los profesionales de mayor edad en situaciones indirectamente asociadas al ejercicio profesional, como la exposición al frío y al calor ambiental (Navarrete et al. Congr.Vet. Panamá 2016). Al igual que en investigaciones anteriores sobre actividades in labore e in itinere, la utilización de algunos EPP fue más frecuente en mujeres que en hombres ${ }^{20}$.

La mayoría de los veterinarios no tiene durante sus actividades equipos completos de EPP ${ }^{10}$. Las correlaciones entre el uso de distintos EPP para una misma actividad fueron escasas o inexistentes, indicando una tendencia a protegerse de manera parcial e incompleta. Esto apoya lo postulado por un autor quien sugirió que los médicos veterinarios son los principales damnificados por el desconocimiento o la falta de observación de normas mínimas de prevención, padeciendo ellos mismos enfermedades vinculadas al manejo de animales ${ }^{2}$.

Asociaciones entre percepción de riesgos y uso de elementos de protección personal. Aunque muchos de los riesgos para la salud tienen su origen en el comportamiento individual y como consecuencia es el propio individuo quien debe ocuparse de prevenirlos, algunos trabajos sugieren que las asociaciones entre las percepciones de riesgo y el empleo de EPP son muy bajas ${ }^{5,10,20}$.

En la presente investigación, ambas variables no estuvieron significativamente correlacionadas. Algunos autores sugirieron que la decisión de utilizar EPP podría basarse en varios factores, incluyendo las normas sociales, la experiencia personal, la capacitación y podría ser impulsada por los rasgos de personalidad de aceptación de riesgos del trabajador ${ }^{9}$. Aunque el ejercicio de la profesión veterinaria implica asumir riesgos laborales, la percepción de estos riesgos aparece como insuficiente.

Si bien no se puede prevenir lo que se desconoce, una mejor percepción de los riesgos no estuvo necesariamente asociada a la toma de medidas preventivas. Parecería que el accidente se asume como algo natural de ocurrencia inevitable y como tal, que no conlleva a necesidad de cambio de comportamiento alguno. La 
falta de uso de distintos elementos de protección en forma conjunta indica una real inconsistencia en el comportamiento profesional de autoprotección.

Agradecimientos. A la Agencia Nacional de Promoción Científica y Tecnológica, por financiar el trabajo (Riesgos ocupacionales en medicina veterinaria, PICT 14-1556).

\section{REFERENCIAS}

1. Alvarez ET, Peratta D, García M, Cavagión L, Larrieu E, Ferrán A. 2007. Enfermedades y lesiones laborales en médicos veterinarios de Argentina. Cien Vet 9: 49-67.

2. Amador AC. 2006. La prevención de riesgos laborales en el sector agrario de los países latinoamericanos. III Congr. Nac. y I Enc. Iberoam. Prev. Riesg. Labor. Sect. Agroalimentario, Santander, España, p. 37.

3. Angoules AG, Linder T, Vrentzos G, Papakostidis C, Giannoudis PV. 2007. Prevalence and current concepts of management of farmyard injuries. Injury 38: S26-S33.

4. Ashford DA et al. 2004. Adverse events in humans associated with accidental exposure to the livestock brucellosis vaccine RB51. Vaccine 22: 3435-3439.

5. Baker WS, Gray GC. 2009. A review of published reports regarding zoonotic pathogens infection in veterinarians. JAVMA 234: 1271-1278.

6. Barke R, Jenkins H, Slovic P. 1997. Risk perceptions of men and women scientists. Soc Sci Quart 78: 167-176.

7. Bernal M. 2003. Los riesgos biológicos en los trabajadores de la salud. Tribuna Médica 2: 49-56.

8. Chitnavis JP, Gibbons CL, Hirigoyen M, Parry JL, Simpson AH. 1996. Accidents with horses: what has changed in 20 years? Injury 27: 103.

9. Dellavalle CT, Hoppin JA, Hines CJ, Andreotti G, Alavanja MC. 2012. Risk-accepting personality and personal protective equipment use within the agricultural health study. J Agromedicine 17: 264-276.
10. Dowd K, Taylor M, Toribio JA, Hooker C, Dhand NK. 2013. Zoonotic disease risk perceptions and infection control practices of australian veterinarians: call for change in work culture. Prev Vet Med 111: 17-24.

11. Flynn J, Slovic P, Mertz CK. 1994. Gender, race and perception of environmental health risks. Risk Analysis 14: 1101-1108.

12. Grandin T. 1999. Safe handling of large animals. Occup Med 14: 195-212.

13. Hope A, Kelleher C, Holmes L, Hennessy T. 1999. Health and safety practices among farmers and other workers: a needs assessment. Occup Med 49: 231-235.

14. Kriss R, Kriss V. 1997. Equine-related neurosurgical trauma: a prospective series of 30 patients. $J$ Trauma 43: 97-99.

15. OMS. 2002. Informe sobre la salud en el mundo, Capítulo 3: Percepción de los riesgos, p. 31-50. http://www.who.int/ whr/2002/es/

16. Reyes J, Sánchez M, Lotero M, Restrepo M, Palacio L. 2010. Seroprevalencia e incidencia de Brucella sp en vacunadores del programa para el control de brucelosis bovina, en el Depto. Antioquia-Colombia. Rev Colomb Cien Pec 23: 35-46.

17. Schenker MB, Orenstein MR, Samuels SJ. 2002. Use of protective equipment among californian farmers. Am J Ind Med 42: 455-464.

18. Slovic P, Malmfors T, Mertz CK, Neil N, Purchase IF. 1997. Evaluating chemical risks: results of a survey of the British Toxicology Society. Human and Exp Toxicol 16: 289-304.

19. Thomas KE, Annest JL, Gilchrist J, Bixby-Hammett DM. 2006. Nonfatal horse-related injuries treated in emergency departments in the United States, 2001-2003. Brit J Sports Med 40: 619-626.

20. Wright JG, Jung S, Holman RC, Marano NN, McQuiston JH. 2008. Infection control practices and zoonotic disease risks among veterinarians in the United States. JAVMA 232: $1863-1872$. 